Middleton, C. (2010). Delivering Services over Next Generation Broadband Networks. Telecommunications Journal of Australia, 60(4), $59-1$.

Link to the Telecommunications Journal of Australia: http://telsoc.org/ 


\title{
Delivering Services Over Next Generation Broadband Networks: Exploring Devices, Applications and Networks
}

\author{
Catherine A. Middleton
}

Ted Rogers School of Management, Ryerson University, Toronto, Canada

catherine.middleton@ryerson.ca

Paper to be published in the Telecommunications Journal of Australia, November 2010.

Investment in 'next generation' (very high speed) broadband infrastructure is expected to enable access to services that will provide citizens with social and economic benefits. Developing services for access using broadband infrastructure can be quite complicated however. This paper explores how services can be delivered over broadband network infrastructure, outlining four broad categories of access devices, three service delivery platforms and two types of networks. Using Australian data, the paper explores user behaviours and expectations around service delivery, illustrating the need for diversity and choice of service offerings. It also notes the importance of mobile services, and makes the case that wireless broadband connectivity should be part of a national broadband infrastructure.

\section{Introduction}

Broadband networks are widely considered to be the foundation of the digital economy. Often described as the infrastructure of the 21 st century, broadband connectivity is understood to be central in enabling individuals to engage in an information society, and in contributing to economic productivity. The Broadband Commission for Digital Development goes so far as to say that broadband connectivity is becoming "the foundation of public services and social progress" in developed and developing nations (Broadband Commission for Digital Development 2010a).

Broadband connectivity offers a great deal of promise, but the actual mechanisms for creating societal benefits by connecting citizens to broadband networks are not well articulated. Progress has been made in establishing regulatory environments and funding policies for developing broadband infrastructure (e.g. in the EU: European Commission 2009a; European Commission 2009b). Projects like Australia's National Broadband Network (NBN)

(Government of Australia 2009), New Zealand's Ultra-Fast Broadband Initiative (Ministry of Economic Development 2010), and Singapore's Next Generation National Broadband Network (Infocomm Development Authority of Singapore 2010a) are all underway, and will result in nationwide infrastructures that connect citizens to next generation broadband networks.

Proponents of broadband networks as an enabler of economic and social benefits cite a variety of services that such networks will support. For instance, in the glossy brochure accompanying the launch of Australia's NBN, Australians are promised "real time, highdefinition video conferencing", "opportunities for remote patient monitoring", "better access to online curriculum content", and "use of interactive content and enhance[d] remote learning opportunities" (Government of Australia 2009). These themes of improved connectivity, enabling e-health and e-learning opportunities (among others) are echoed throughout the international discourse on the benefits of broadband (Broadband Commission for Digital 
Development 2010b; Department for Culture Media and Sport and Department for Business Innovation and Skills 2009; Ezell et al. 2009; Federal Communications Commission 2010; i2 Media Research 2010; Organisation for Economic Co-Operation and Development 2008; 2009).

But broadband networks are just conduits. The next generation network infrastructure being built in various countries is exactly that, infrastructure. To create value for users and realise the promises of network promoters, fibre optical or wireless 'pipes' must be connected to users' devices, and services must be available for delivery over the network. While this observation is obvious to many, there are many complexities inherent in developing broadband services (applications/content) to be delivered over a national infrastructure to a variety of access devices. These points are explored below, with a focus on services used by individual citizens, not by businesses. ${ }^{1}$

\section{Broadband Users and Access Devices}

The Australian Bureau of Statistics (ABS) (2009) reported that $62 \%$ of Australian households had a broadband connection, and the Digital Futures project (Ewing and Thomas 2010) estimated broadband uptake at $75 \%$ in 2009 . As such, somewhere between a quarter and a third of Australian households do not currently subscribe to a broadband service. The ABS also noted that $22 \%$ of Australian households did not have access to a computer in 2009. Until quite recently, it was difficult to access broadband services without a computer, but the nature of computers is changing, resulting in a broader range of connectivity options. Additionally, some broadband services (e.g. videoconferencing) can be accessed without a computer. Not having a computer no longer excludes a household from accessing broadband services, provided that the services are designed for delivery to other devices that might be available to household members, or that potential users access a broadband connection at another location (e.g. workplace, public library). Indeed, with more than a fifth of the population not having a home computer, alternative means for providing citizens with access to broadband services are essential.

There are four broad categories of devices that can be used to access broadband applications and services. An increasingly popular means of accessing broadband services is by use of a smartphone, and more recently, a tablet computer (e.g. Apple's iPad). Portable computers and desktop computers are still the most common access devices, and are considered in separate categories. A less common option, one that does not provide internet access but does allow access to some broadband services is the combination of an internet protocol (IP) gateway (e.g. a set-top box) and a consumer device (e.g. television or computer monitor, telephone). Each of these is discussed below.

\section{SMARTPHONES/TABLETS}

Smartphones are mobile phones with embedded computing power. To access broadband services, the important functionality in a smartphone is the data connection (enabling network access), not the mobile phone. For this reason, devices like Apple's iPod Touch (a handheld mobile computing device without a phone) can be included in this category. Smartphones and tablets (a new form factor popularised by the Apple iPad, offering mobile data access and

\footnotetext{
${ }^{1}$ Although this paper does not address business to business applications, their importance should not be overlooked when assessing the value of national broadband infrastructure. It also does not consider narrowband applications like telemetry that will be an essential part of a national information infrastructure.
} 
services on a larger device than a smartphone) use different operating systems than computers, typically running applications ('apps') rather than the standalone software programs that are installed on computers. There are differences in the functionality of smartphones and tablets. For instance, screen size is an important differentiator - tablets are better suited to watching video, web browsing and reading/editing documents. Both use wireless connections (Wi-Fi or $3 \mathrm{G}$ ) to access content and services. These devices are popular because they are small, highly mobile and support multiple activities (including entertainment, communication, and information seeking).

More than $20 \%$ of mobile phones in the US are smartphones (Comscore 2010). Gartner (2010) forecasts that 268 million smartphones will be sold globally in 2010, rising to more than 875 million in 2014. Once a luxury item, smartphones are becoming more affordable. As individuals replace their current mobile phones, they will be encouraged to switch to smartphones (which provide greater revenues to mobile carriers), and in a few years it is expected that the majority of mobile phones will be smartphones. While many smartphones are currently used primarily for text and voice connectivity, they do provide users with the capacity to access broadband services and to run applications, activities that are expected to grow in popularity over time.

The iPad tablet has been criticised for limited functionality (e.g. no USB port for file transfers, no camera or support for videoconferencing, unable to run Flash video or multiple applications at once) yet Apple sold more than 3 million iPads in the first three months of sales (Apple 2010). iPad sales are insignificant in comparison to smartphone sales, but the iPad marks the arrival of the tablet as a viable consumer device. A variety of tablets running competing operating systems are now coming to market, and they will likely offer much of the functionality missing from the first generation iPad. It is estimated that by $2015,23 \%$ of personal computers sold in the US will be tablets (Epps 2010).

In the context of providing broadband services, smartphones and tablets are important because they are relatively cheap access devices that have broad market appeal. With touch screen interfaces and 'point and tap' applications, these devices can be less intimidating for new users than traditional PCs, and may help encourage those who are currently not accessing any broadband services to get connected. Fibre to the premises (FTTP) networks do not provide direct broadband connectivity to these devices, although users can create wireless networks within their homes to provide access. At present there are no wholesale wireless networks that service providers can use to connect to tablet and smartphone users (but the devices have the advantage of being able to connect to $3 \mathrm{G}$ mobile broadband networks when Wi-Fi is unavailable). Additionally, most services that currently work on portable or desktop computers will need to be repurposed or modified for delivery to these new mobile devices. Although smartphones and tablets are becoming increasingly popular, it may not be easy to deliver broadband services to them.

\section{PORTABle COMPUTERS}

This category includes laptop, notebook and netbook computers. By 2015, laptops (also called notebooks - fully featured portable computers, with similar functionality to desktop computers) are expected to make up $42 \%$ of personal computer sales in the US. Netbooks (smaller and lighter computers, with less power and functionality than desktops or laptops) are forecast to be the choice of 17\% of American computer purchasers in 2015 (Epps 2010). Collectively, these devices offer more functionality than tablets and smartphones (e.g. improved capacity for creating content, ability to run traditional software programs, more 
flexible file management and improved document editing), but do not have the same userfriendly interface nor do they run apps. They can connect wirelessly to broadband networks using $3 \mathrm{G}$ or Wi-Fi, and can also connect through a wired home network to the fibre-to-thepremise FTTP networks being built as part of national next generation broadband projects.

Portable and tablet computers may converge over time, resulting in affordable portable devices that provide more options for managing files and creating content, while also offering touch screen interfaces and supporting apps as well as traditional software programs. In their current form, portable computers will be used from multiple locations, using connections that may or may not make use of FTTP to access broadband services through traditional software interfaces (e.g. web browsers, email clients, file sharing services, commercial music and video providers).

\section{DESKTOP COMPUTERS}

Desktop computers, designed for use in fixed locations, are expected to account for just $18 \%$ of new computer purchases in the US by 2015. Even in 2015 however, desktop computers are still expected to be more common than tablets, laptops or netbooks (Torrens 2010). Desktop computers are likely to be used in individuals' homes, where they can take full advantage of wired connectivity.

The sorts of broadband services envisaged to provide social and economic benefits to citizens can be delivered easily to desktop PCs, but some functionality may be lost because the PCs are connected in a fixed location. For households with a single PC shared among users, a decision must be made as to where the computer should be physically located to provide optimal access for a variety of services. Some services might be best suited to a private location, while others might be more useful in a easily accessible common space.

\section{FiXed LINE ACCESS WITHOUT A COMPUTER}

In many countries, internet adoption rates appear to be plateauing at about $75-80 \%$ of the population (with broadband adoption rates somewhat lower). There are a variety of reasons that people choose not to connect to the internet, including cost, perceived complexity, and lack of interest (Dailey et al. 2010; Horrigan 2010). Older individuals, and/or those with low incomes or less education are least likely to be internet users (Australian Bureau of Statistics 2009; Ewing and Thomas 2010), but like the rest of the population, these individuals could benefit from services delivered using broadband networks.

As noted, broadband networks can deliver services without an internet connection, simply using a managed internet protocol (IP) network (the NBN Implementation study refers to these as 'direct delivery' or 'standalone' services, McKinsey \& Company and KPMG 2010). Networks like Australia's National Broadband Network will bring a fibre optic connection to a household. A service provider can then connect the fibre network to peripherals like a television screen and IP camera for use in videoconferencing, or a voice over IP (VoIP) telephone. The network can also support smart grid services (e.g. to enable monitoring and control of electrical devices), telehealth applications (e.g. transferring medical data between a household and a healthcare provider) and many others. This sort of functionality is not yet common in households, but applications like videoconferencing and VoIP telephony are widely used in the public sector (see Alberta SuperNet 2006, for a description of how an IP network enables broadband service provision throughout the Canadian province of Alberta). 
Even without a computer, households can access some of the benefits offered by broadband networks. IP-based managed services are different than the software or app-based services accessible on computers though, and require separate development. Managed services can be offered to anyone connected to a FTTP network, and have the potential to be especially valuable in those households that do not have a personal computer connected to the internet. With more than $99 \%$ of metropolitan Australian households having at least one television set (Screen Australia 2009), there is potential to provide some broadband services to almost all Australians using their television set as an access device. Of course, the television set can also be connected to a broadband network as a television, enabling interactivity and content on demand in addition to traditional television programming.

\section{USER BEHAVIOURS}

Many Australians are already using multiple devices to access broadband services, and for those who are not yet connected there are now simple options available. Smartphones and tablets have uncomplicated user interfaces, and built-in connectivity (when $3 \mathrm{G}$ access is enabled), making them easier for most to use than personal computers. The option of delivering services over managed IP networks to familiar devices like telephones and TVs also reduces the complexity of using a broadband network. Services are not free, meaning that cost may still be an access barrier, and those who are not currently internet users may not be aware of the potential benefits of broadband connectivity. Given the range of devices and connectivity options now available however, broadband access of some sort should be available to everyone who wants it, creating the possibility that widespread social and economic benefit can be realised from investment in a national broadband infrastructure.

That said, to date broadband networks are primarily used for communication and entertainment purposes, with services delivered over the internet (see Ewing and Thomas 2010, for data on internet use in Australia). Individuals do use broadband to access government and health information, and for educational purposes (e.g. online research), but extensive e-health, e-learning and e-government services that promise notable social and economic benefits are not yet well-established or widely available in broadband economies around the world. As part of the development of the Singaporean Next Generation National Broadband Network, a program has been created to support the development of innovative services that make effective use of higher bandwidth connectivity (Infocomm Development Authority of Singapore 2009) and a demonstration site (the iExperience Centre) is now showcasing next generation services. The NBN Implementation study recognises the potential for advanced services delivery over the NBN, but such services are not yet common (McKinsey \& Company and KPMG 2010).

\section{Applications and Services}

National broadband infrastructures that offer wholesale network access enable a wide range of service providers to use these networks to connect to citizens. The focus here is on services available to individuals (as opposed to enterprise services), but detailed consideration of how service providers actually connect to national broadband infrastructure is beyond the scope of this paper. The discussion here assumes that service providers can negotiate access to the network, and that they have the freedom to develop and deliver innovative services that will meet the needs of their users. Service providers are often referred to as 'retail service providers,' a term that encompasses internet service providers (ISPs) as well as other entities that sell services. The focus here is not on internet service provision (an essential part of a national broadband infrastructure), but on the organisations and individuals that will provide 
services (entertainment, education, health, communication, e-government etc.) used on the network.

Services are essential in enabling users to benefit from broadband access, but there is enormous complexity inherent in developing services that are accessible to users on the device(s) that they prefer. As the previous section notes, many users have adopted multiple devices, but others may use only one. Service providers are faced with decisions as to how to offer their services, and may need to develop expertise across a variety of access platforms. The discussion distinguishes 'over-the-top' services from 'managed' services, notes the challenges in developing mobile applications, and discusses the need for hardware to enable delivery of some services.

\section{OVER-THE-TOP SERVICES}

At present, the majority of broadband users rely exclusively on over-the-top (OTT) services. This terminology is used to describe services that are delivered over the internet, on a 'best effort' basis. In contrast, managed services require enhanced quality and/or security, and are delivered on managed IP networks, for instance with quality of service (QoS) protocols enabled (see McKinsey \& Company and KPMG 2010, on this point). Managed services can operate on a standalone basis, meaning that an individual does not need an internet connection for access.

The simplest OTT services are offered through a web browser. To access a service, a user simply enters the URL in a browser, and then engages with the website. Web-based services are effective for searching, information dissemination, file sharing, accessing audio or video content, and transactions (online banking, bill payment), and are familiar to most users accessing the internet using a PC. Websites are relatively easy to design and maintain, and will continue to be a key element for service delivery in the foreseeable future.

Services can also be provided over the internet using software installed on users' computers. For instance, programs like Skype or Google Voice allow people to talk 'on the phone' to each other, messenger programs enable instant chatting, and entertainment platforms like iTunes provide access to music, movies, television and books. Online games like World of Warcraft allow individuals to interact with each other, and virtual worlds like Second Life can be used for entertainment but also for e-learning or to provide e-health services (e.g. support groups). Developing software to support a service is more complex than developing a website, but allows for a much richer interface and user experience. Programs like these could be adapted or extended to offer a broader range of services.

OTT services work well for those accessing the internet using a personal computer. It is possible to access websites with a smartphone, but the experience is often sub-optimal (many phones do not support scrolling effectively, it can be difficult to click on embedded links, and the screen size limits the amount of text that can be displayed). The experience is somewhat improved on tablet devices, but mobile phones and tablets often don't have the necessary software to handle downloaded files, or to display online content (e.g. video). Some users find that it is difficult to enter data onto a website using a mobile device, and the cost of accessing services using a mobile device (smartphone or tablet) can be prohibitive.

\section{MOBILE APPS}

Apple's iPhone had a big impact on the smartphone market, popularising the concept of apps, and improving mobile web browsing. Apps are self-contained applications for mobile 
devices. Designed specifically for a particular mobile operating system, apps provide access to an enormous range of services and content. Apps are designed to advantage of the specific functionality present in a particular smartphone. Apps can make use of GPS/location data to provide location specific services, can synchronise information between computers and mobile devices, and can be customised more easily than websites (e.g. allowing users to choose the types of news stories they want to highlight in a newspaper app). A wide range of businesses, public sector agencies (e.g. transit authorities, local governments) and individuals have created apps delivering value to mobile device users. Many apps are available free of charge, and those that are for sale often cost just a few dollars.

At present, five operating systems dominate the smartphone market. Nokia's Symbian is the global market leader ( $40 \%$ market share), RIM's BlackBerry OS and Google's Android are competing for second place $(\sim 17-18 \%$ each), followed by Apple's iOS $(\sim 15 \%)$ and Microsoft's Windows Mobile (<7\%) (Gartner 2010; IDC 2010). In Australia in Q1 2010 Symbian had $45 \%$ market share, followed by Apple iOS with $40 \%$, BlackBerry with $9 \%$ and Android with 2\% (Colley 2010). This market is very dynamic, and it is possible that some of these operating systems may disappear over the next few years if/as the market consolidates. From the perspective of delivering next generation broadband services, recognising multiple operating systems (OS) is important, because the OS are incompatible. An application developed for use on Apple's iOS will work on an iPhone, iPod Touch or iPad, but not on a Nokia or BlackBerry device.

The operating systems used for smartphones also power tablet computers. Some smartphone applications will run on tablets using the same OS, but others need to be modified to fit the tablet form factor, meaning additional development may be needed to provide services to both devices. At the time of writing, Apple's App Store offered about 250,000 apps, compared to about 100,000 for Google's Android platform. Microsoft, Symbian and RIM do not have well-developed apps markets, but arguably what is important is that each platform has a sufficient number of effective, useful applications to meet user' needs.

Apps take advantage of the touch interface found on many smartphones, making them very easy to use. For someone who is unfamiliar with a computer interface, it is much easier to access web content (e.g. online newspaper, social networking sites, online video) through an app than a web browser. Rather than using a mouse to launch a web browser and then navigating to the appropriate site, a user simply touches the app (easily identifiable with an icon) and the content appears on the screen.

Apps change the dynamics of internet access, moving away from a browser-based routine to provide users with a richer experience. With five operating systems to develop for, and five app stores to negotiate with to make an app available, the complexity of service provision is increased. Additionally, as will be discussed below, broadband content (app- or browserbased) delivered to a mobile device may be transmitted over a mobile operator's network, not a national broadband infrastructure. This changes the economics of the transaction.

\section{Managed Services}

Managed services operate independently of the internet. Services delivered directly to end users via a managed IP network require a different set of competencies in a service provider than over-the-top internet services. Managed services require direct contact with the user to enable the service (e.g. physically connecting hardware to the FTTP network in an 
individual's home). Managed services cannot be delivered over commercial mobile broadband networks (at least not without negotiation with the network operator).

There is great scope for the development of managed services to support health and learning (and managed services are also used to provide entertainment content, e.g. IPTV). Applications like videoconferencing work much better when quality of service protocols are in place, and a direct network connection can also be used for telepresence (an enhanced form of videoconferencing), running software, sharing large files (e.g. educational videos or medical images), and monitoring applications. Hardware can be integrated to provide diagnostic capabilities (e.g. blood pressure or blood sugar device monitoring). Because managed services do not require an internet connection they can be delivered to people without computers, extending the reach of services to a population that could not previously benefit from broadband connectivity. With the appropriate user interface, managed services can be easy to use (e.g. controlled through a television, or through a dedicated device with a simple operating controls, perhaps analogous to a household appliance). There will likely be a sizeable group of users whose only broadband services are delivered through a managed IP network, but many others will combine managed services with OTT internet services and/or mobile broadband access.

\section{IMPLICATIONS FOR SERVICE PROVIDERS}

FTTP networks work well to deliver services over the internet, and through managed networks. The rise of mobile services adds a level of complexity. Citizens are not likely to be too aware of the differences between their mobile broadband provider and the ISP from whom they get access to a FTTP network. Mobile broadband users will understand that there is a different pricing structure for mobile services, but could reasonably assume that most services will be available on whatever platform they choose to use.

The discussion above indicates that services must be built in different ways for use on different devices. It is quite possible that a single provider could be expected to offer a service through a software or web interface (OTT), as well as making it available for mobile browser, and for all five different mobile operating systems. This increases the cost of service development, and may stretch the resources of the service provider. The proliferation of service delivery platforms may also require that organisations change their service delivery processes and policies, a point that is noted by McKinsey \& Company and KPMG (2010) when discussing the challenges of implementing e-government services on Australia's NBN.

\section{Broadband Networks}

Many national broadband networks are designed as 'open access' networks, meaning that multiple service providers can offer services to a household using a single broadband connection to the home. While these networks may or may not be owned by national governments, they are built in part with government funds, and are managed in a way to provide public benefit. Service providers negotiate network access with the wholesale operator (NBN Co in Australia), and then make direct arrangements with the household to provide specific services. For instance, a household might choose to purchase internet access from one company and television service from another, as well as selecting several government services offered through a combination of OTT and managed access. Open access fosters competition among service providers, encourages innovation and allows citizens choice in their service providers. Additionally, the open access/wholesale model is designed to ensure affordability of network access (for service providers and end users), as the connection to the home is not controlled by a monopolist operator. 
In Australia, it is anticipated that $93 \%$ of premises can be connected to the National Broadband Network using fibre (FTTP). For premises that choose to connect, the fibre will enter the home at a single point, and can be connected to an existing in-home wired network, or to a wireless router to create an in-home wireless network. Laptops and desktops can be connected to wired or wireless (Wi-Fi) in-home networks, and mobile devices can use the wireless network.

It is important to differentiate between wireless connectivity that is provided through a national broadband network and that which is provided by mobile operators (e.g. $3 \mathrm{G}$ networks providing data access for smart phones, or USB/dongle mobile broadband connections for laptops). Mobile providers operate their networks on a commercial basis, and are not subject to open access regulation. Users have a choice of mobile providers, but there is no guarantee that a service provider that doesn't own a network (e.g. government agency) can ensure affordability of its services when accessed through a commercial mobile network. The Singaporean national broadband network does include a wireless service as part of its national infrastructure (Infocomm Development Authority of Singapore 2010b). Elsewhere however, next generation broadband developments are currently focused on providing FTTP connectivity (offering wireless or satellite connectivity only where it is uneconomical to lay fibre).

Mobile internet access devices are proliferating - it is not uncommon for technologically savvy consumers to have a smartphone, tablet and laptop computer, frequently coupled with a mobile broadband subscription. In Australia, as of December 2009 35\% of broadband subscriptions were for wireless services (Australian Bureau of Statistics 2010), and Ewing and Thomas (2010) report that 35\% of Australians have accessed the internet using a mobile device. There will be some overlap among mobile broadband subscribers and those who use mobile phones to access the internet, but collectively these numbers suggest that mobile access to broadband applications and services is popular among Australians.

Wireless networks ( $3 \mathrm{G}$ or Wi-Fi) are capable of supporting most of the applications and services on the market today (see Williamson 2010 for a detailed discussion of the value of wireless networks). Although priced at a premium, mobile services are convenient and offer value to their users. While it is unlikely that wireless networks alone will be able to support full range of next generation services envisaged by national broadband plans (Tucker 2010), it is easy to understand why individuals want the flexibility and convenience afforded by mobile services. As more services that provide broader social benefits are developed (egovernment, e-health, e-learning etc.), it is perfectly reasonable for citizens to expect these services to be available to them via wired or wireless networks.

Users are looking for seamless engagement with broadband services, and many are unaware of the differences in access arrangements between open access fibre networks and commercial mobile networks. If national governments want to ensure the broadest possible access to, and take up of, next generation broadband services, delivery only to homes connected to fibre networks will be insufficient. Development of open access wireless infrastructures could facilitate service innovation, but would require significant change in current industry structures. Nevertheless, as recognised by the FCC in the American national broadband plan (Federal Communications Commission 2010), the development of good wireless infrastructure is a policy objective that should be pursued. Ultimately, a combination 
of FTTP, Wi-Fi and 3G/4G networks should be deployed to meet citizens' connectivity needs.

\section{Discussion}

In a promotional document for the NBN (Government of Australia 2009, p. 1) it is noted that "to get the best out of the new opportunities offered by the internet, people need to be able to upload and download information." This paper demonstrates that this is a rather narrow view of next generation broadband infrastructure. Downloading is certainly a valid way to disseminate information, but it is one of many. Although the internet will be central to many broadband services, internet connectivity is not an essential component for service delivery. Initial visions of service delivery via the NBN seem to centre on individuals accessing the NBN on their home computers, but there are many other possibilities.

Different users will have different needs. Demographics and geography influence demand for services and choice of access device(s). Service providers can reach new users (bridging the digital divide to reach those who are uncomfortable with computers) by offering services on mobile devices or direct to appliances in the home. Many users are familiar with multiple means of accessing broadband networks, and expect services to be available on the platforms of their choice. Given the diversity of platforms however, it may not be economical to serve them all. Service providers must make difficult strategic decisions about how to best delivery value to end users, in a context of rapid technological change. This paper has outlined components involved in such decision making.

Broadband networks have great potential for delivering innovative services to provide social and economic benefit. To make this happen, service providers must carefully assess the needs and expectations of users, and develop affordable, accessible services that provide value to users on devices that they choose, wherever they want to be. In particular, delivering services to wireless devices, allowing users mobility and the added functionality of location-based services, is essential to realising tangible benefits in the long term.

\section{Closing Comments}

This paper offers insight into the complexities of developing next generation broadband services in ways that will create social and economic benefits for users. It highlights the need for service developers to understand their potential customers, and to think carefully about which platforms are best suited for service delivery. It demonstrates the relationships among users, devices, services and networks, illustrating why an understanding of these interconnections is important for developing services that will meet the needs of a diverse population of users.

This discussion focused on Australia's National Broadband Network, but the elements of service development and delivery described here are common to other national broadband infrastructure projects. The nature of access devices, methods of service delivery, types of services and user preferences will change over time, but the complexity of the multi-device, multi-service, multi-user environment will remain. It is likely that demand for wireless broadband will continue to grow, creating pressure to incorporate wireless networking into national broadband infrastructures. Policy makers and service providers are advised to keep close watch on, and to respond to changes in user behaviours, access technologies and network usage patterns when creating an environment in which broadband services can bring real benefits to citizens. 


\section{References}

Alberta SuperNet. 2006. 'Harnessing the SuperNet Advantage'. [Internet]. Accessed 11 September 2010. Available from: http://www.axia.com/documents/networks/AlbertaVenture SNet.pdf.

Apple. 2010. 'Apple Reports Third Quarter Results'. [Internet]. Accessed 5 September 2010. Available from: http://www.apple.com/pr/library/2010/07/20results.html.

Australian Bureau of Statistics. 2009. 'Household Use of Information Technology, 20082009'. Canberra: Australian Bureau of Statistics.

Australian Bureau of Statistics. 2010. 'Internet Activity, Australia, December 2009 - Internet Subscribers by Access Connection, for ISPs with More Than 1,000 Active Subscribers'. Accessed 30 August 2010. Available from:

http://www.abs.gov.au/AUSSTATS/subscriber.nsf/log?openagent\&81530do001 200912.xls $\& 8153.0 \&$ Data\%20Cubes\&6F717ADDA2F1D1F0CA2576F50011B2C7\&0\&Dec\%202009 \&30.03.2010\&Latest.

Broadband Commission for Digital Development. 2010a. 'About the Broadband Commission'. [Internet]. Accessed 3 September 2010. Available from: http://www.broadbandcommission.org/about.html.

Broadband Commission for Digital Development. 2010b. 'What Broadband Brings'.

[Internet]. Accessed 3 September 2010. Available from: http://www.broadbandcommission.org/whatbroadbandbrings.html.

Colley, Andrew. 2010. 'Surging iPhone Hot on the Heels of Nokia as Australia's No 1 Smartphone'. The Australian. Accessed 15 September 2010. Available from:

http://www.theaustralian.com.au/australian-it/surging-iphone-hot-on-the-heels-of-nokia-asaustralias-no-1-smartphone/story-e6frgakx-1225879621669.

Comscore. 2010. 'Comscore Reports May 2010 U.S. Mobile Subscriber Market Share'.

[Internet]. Accessed 11 September 2010. Available from:

http://www.comscore.com/Press Events/Press Releases/2010/7/comScore Reports May 2010 U.S. Mobile Subscriber Market Share.

Dailey, Dharma; Bryne, Amelia; Powell, Alison; Karaganis, Joe; Chung, Jaewon. 2010.

'Broadband Adoption in Low-Income Communities'. Brooklyn, NY: Social Science

Research Council.

Department for Culture Media and Sport; Department for Business Innovation and Skills. 2009. 'Digital Britain - Final Report June 2009'. Kew, Richmond, Surrey: Office of Public Sector Information.

Epps, Sarah Rotman. 2010. 'The US Consumer PC Market in 2015'. Cambridge, MA:

Forrester.

European Commission. 2009a. 'Community Guidelines for the Application of State Aid Rules in Relation to Rapid Deployment of Broadband Networks'. [Internet]. Accessed 10 September 2010. Available from:

http://ec.europa.eu/competition/consultations/2009 broadband_guidelines/guidelines en.pdf.

European Commission. 2009b. 'Draft Commission Recommendation on Regulated Access to

Next Generation Access Networks (NGA)'. [Internet]. Accessed 10 September 2010. Available from:

http://ec.europa.eu/information_society/policy/ecomm/doc/library/public consult/nga 2/090611_nga recommendation_spc.pdf.

Ewing, Scott; Thomas, Julian. 2010. 'CCi Digital Futures 2010: The Internet in Australia'.

Melbourne: ARC Centre of Excellence for Creative Industries and Innovation,

Swinburne University of Technology.

Ezell, Stephen; Atkinson, Robert; Castro, Daniel; Ou, George. 2009. 'The Need for Speed: The 
Importance of Next-Generation Broadband Networks'. Washington, DC: The Information Technology and Innovation Foundation.

Federal Communications Commission. 2010. 'Connecting America: The National Broadband Plan'. Washington, DC: FCC.

Gartner. 2010. 'Gartner Says Android to Become No. 2 Worldwide Mobile Operating System in 2010 and Challenge Symbian for No. 1 Position by 2014'. [Internet]. Accessed 11 September 2010. Available from: http://www.gartner.com/it/page.jsp?id=1434613.

Government of Australia. 2009. '21st Century Broadband'. [Internet]. Accessed 10 September 2010. Available from:

http://www.dbcde.gov.au/ data/assets/pdf file/0005/110012/National_Broadband N etwork policy brochure.pdf.

Horrigan, John B. 2010. 'Broadband Adoption and Use in America - OBI Working Paper Series No. 1'. Washington, DC: Federal Communications Commission.

i2 Media Research. 2010. 'Next Generation Services for Older and Disabled People'. London: Ofcom.

IDC. 2010. 'Worldwide Converged Mobile Device Market Projections Raised 10\% for the Year'. [Internet]. Accessed 11 September 2010. Available from: http://www.idc.com/getdoc.jsp?containerId=prUS22486010.

Infocomm Development Authority of Singapore. 2009. 'Public Briefing: Next Gen Services Innovation Programme (Ngsip) 1st Call-for-Proposal'. IDA. Accessed 12 September 2010. Available from: http://www.ida.gov.sg/images/content/Infrastructure/nbn/images/pdf/1stBriefing.pdf.

Infocomm Development Authority of Singapore. 2010a. 'What Is the Next Generation Nationwide Broadband Network (Next Gen NBN)?'. [Internet]. Accessed 1 September 2010. Available from: http://www.ida.gov.sg/Infrastructure/20090717105113.aspx.

Infocomm Development Authority of Singapore. 2010. 'Fact Sheet: Wireless@SG'. Accessed 1 September 2010. Available from: http://www.ida.gov.sg/doc/News\%20and\%20Events/News and Events_Level2/2009072816 5354/WirelessSG factsheet.pdf.

McKinsey \& Company; KPMG. 2010. 'National Broadband Network Implementation Study'. Accessed 6 May 2010. Available from: http://data.dbcde.gov.au/nbn/NBNImplementation-Study-complete-report.pdf.

Ministry of Economic Development. 2010. 'Ultra-Fast Broadband Initiative'. [Internet]. New Zealand Government Accessed 11 September 2010. Available from: http://www.med.govt.nz/templates/ContentTopicSummary 41902.aspx.

NBN Co Limited. 2010. 'Broadband Made Easy'. [Internet]. Accessed 8 September 2010. Available from: http://www.nbnco.com.au/about-nbn-co/nbnco-broadband-madeeasy.

OECD Directorate for Science Technology and Industry. 2009. 'Network Developments in Support of Innovation and User Needs'. Paris: OECD Working Party on Communication Infrastructures and Services Policy.

Organisation for Economic Co-Operation and Development. 2008. Broadband Growth and Policies in OECD Countries. Paris: OECD.

Prime Minister of Australia. 2009. 'New National Broadband Network'. [Internet]. Government of Australia. Accessed 10 September 2010. Available from: http://www.minister.dbcde.gov.au/media/media releases/2009/022.

Screen Australia. 2009. 'Proportions of Australian Homes with Television Sets, Metropolitan Markets, 1997-2009'. Accessed 11 September 2010. Available from: http://www.screenaustralia.gov.au/gtp/wftvviewtvs.html. 
Torrens, Herb. 2010. 'Report: Tablets to Grab Market Share over Netbooks'. The Journal, 26 July.

Tucker, Rodney S. 2010. 'Broadband Facts, Fiction and Urban Myths'. Telecommunications Journal of Australia 60 (3): 43.1-43.15.

Williamson, Brian. 2010. 'Nomadicity and the Evolution of Applications, Networks and Policy'. Telecommunications Journal of Australia 60 (4).

\section{Acknowledgement}

This research was supported by a Social Sciences and Humanities Research Council of Canada grant: Developing Next Generation Broadband Infrastructure: Learning from Australia's National Broadband Network (Investigators: Catherine Middleton, Ryerson University, Canada, and Jock Given, Swinburne University of Technology, Australia). 